que la courbe se traçait; d'autre part, puisque la hauteur $h$ d'où on a lâché le poids est connue ainsi que la longueur $A B$, on connaît toutes les circonstances du mouvement vertical entre $\mathrm{A}$ et $\mathrm{B}$ et, en particulier, la durée nécessaire pour parcourir cet espace ou pour tracer la courbe. On peut donc avoir sans difficulté la vitesse angulaire du disque et l'exprimer en nombre de tours à la minute.

Cet appareil répond bien ainsi à l'objet que s'était proposé l'auteur et il serait facile de montrer que l'approximation probable avec laquelle le résultat est acquis est, en mettant tout au pire et en opérant sans le moindre soin, voisine de 3 pour roo; et que, pour peu qu'on veuille s'en donner la peine, on la peut réduire aisément au $1 / 3$ de cette valeur, soit I pour roo.

Mais il se trouve, circonstance heureuse, que cet appareil si simple permet de mesurer, avec une approximation égale, l'écart entre la vitesse réelle de rotation du disque à chaque instant et la vitesse constante avec laquelle il devrait tourner pour décrire d'un mouvement uniforme l'arc A A' B B' A A', pendant que le poids tombant de la hauteur $h$ parcourt la longueur $\mathrm{AB}$.

Rien n'est plus simple, en effet, que de calculer cette vitesse angulaire constante. Elle est égale à l'arc A A' B B' A A' divisé par la durée du passage devant AB. Autant de fois cette vitesse sera contenue dans l'arc total, autant d'époques différentes on pourra considérer dans la durée du passage et, par suite, autant on pourra marquer sur $A B$ d'abaissements au-dessous du point $A$ faciles à évaluer.

Le travail ainsi préparé, une construction géométrique très simple et très facile à exécuter sur le disque lui-même pris comme planche à dessin, permettra de tracer la courbe que le pinceau eût dû marquer sur le disque si le mouvement de celui-ci avait été uniforme (traits ponctués de la figure ci-jointe).

La comparaison d'abaissements simultanés lus sur l'une ou l'autre courbe permet alors de voir si, à toute époque, le disque était en avance ou en retard sur son mouvement moyen, et de combien; par suite d'évaluer les différences et les rapports des vitesses angulaires a chaque instant. C'est proprement la mesure du coefficient d'irrégularité, mesure obtenue par des procédés graphiques simples et avec une approximation adéquate à celle que nous avons signalée plus haut.

L'appareil de M. le commandant Audebrand est inspiré des dispositions que Morin a adoptées, sur le conseil de Poncelfet, pour la mesure des accélérations dans ses recherches sur le frottement; mais notre auteur substitue au mouvement d'horlogerie, dont faisait usage le savant Général, le poids même dont celui-ci s'est ultérieurement servi de manière si ingénieuse pour faire inscrire par un grave la loi de sa chute sur un appareil désormais classique. A notre connaissance, cette combinaison et cette adaptation des deux appareils n'avair pas encore été faite.

Nous croyons savoir que $M$. le commandant Auderrand se propose de faire une communication détaillée au sujet de son appareil au prochain Congrès de l'Ássociation française pour l'Avancement des Sciences, qui se tiendra au mois d'août prochain à Grenoble, et qu'il a l'intention de le soumettre aussi au jugement de plusieurs sociétés savantes et industrielles. Les électriciens étant à la recherche d'appareils simples et précis permettant de mesurer les moindres variations de vitesse angulaire, nous ne doutons pas quils fassent le meilleur accueil à l'ingénieux procédé de notre collaborateur.

E.-F. Côte.

\section{RÉSERVOIR EN CIMENT ARMÉ}

Jusqu'en juin rgo3, la ville de Tucuman, chef-lieu de la province du même nom (République Argentine), était alimentée en cau par un ancien réservoir en maçonneries, de forme rectangulaire et d'une capacité de 4 ooo mètres cubes. D'importantes fuites s'étant déclarées à travers des fissures qui s'étaient produites dans les maçonneries de ce réservoir, on fut obligé de réduire de moitié la hauteur de l'eau, ce qui entraîna en même temps une réduction de moitié dans la capacité. Enfin, ces fuites n'ayant pu être aveuglées, on résolut de construire un nouveau réservoir et l'on profita de l'occasion pour porter sa capacité à 5000 mètres.

Les filtres et l'ancien réservoir se trouvaient tout près de la ligne à double voie du Ferrocaril Central del Norte; aussi a-t-on accusé les trépidations produites par le passage des trains de n'être pas tout à fait étrangères à la formation des fissures des maçonneries qui avaient été construites un peu trop légèrement, comme l'a montré la vérification des calculs de stabilité. De plus, les fondations reposaient sur un terrain marneux assez résistant à sec mais qui, devenant plastique sous l'action de l'eau, devait perdre de plus en plus ses propriétés de résistance à la compression sous l'action des infiltrations, toujours possibles par suite de la non complète perméabilité des maçonneries, surtout au début.

Lorsque la création d'un nouveau réservoir fut chose dé. cidée, les pouvoirs publics firent étudier divers projets afo de rechercher quel serait le meilleur système à employer. Et tout d'abord, afin de n'avoir pas à faire une nouvelle et coll. teuse installation de prises d'eau, de filtres et de canalisa. tions, on résolut de construire le nouveau réservoir à proxi. mité de l'ancien; on fut donc amené à rechercher quel serait le meilleur système qui serait à la fois assez élastique pour atténuer l'effet des vibrations et assez imperméable pour n'avoir pas à craindre un affaiblissement du sous-sol. Un réservoir métallique aurait bien réalisé ces conditions, mais l'on craignit que la chaleur humide des tropiques, sous les. quels Tucuman se trouve presque, ne vînt à ronger rapidement les tôles sous l'action d'une oxydation énergique. Le réservoir précédent qui était en maçonneries ordinaires ayant donné de mauvais résultats, on évita de recommencer l'expérience; par contre le ciment armé parut tout indiqué. En effet, grâce à l'armature métallique intérieure, il permettait de faire de l'ensemble un tout assez rigide pour assurer une assise meilleure qu'avec la maçonnerie ordinaire, tout en étant assez élastique contre les trépidations. De plus, il permettait de réaliser, sur les autres procédés, une assez sérieuse économie; sur un réservoir en maçonnerie-les projets laissaient prévoir un bénéfice de 28 pour cent; c'est à lui qu'ori donna la préférence. Les travaux furent confiés à M. Julio 
Traverse, qui avait déjà étudié et entrepris plusieurs travaux du même genre, sous la direction de $M$. Carlos Wacters, directeur au département des Travaux publics et supérintendant au département des Irrigations, et à l'obligeance de qui nous devons les renseignements suivants.

La figure I montre la disposition générale du réservoir qui a une forme cylindrique. Sẹs parois travaillent seulement à latraction dans le sens horizontal sous l'action de l'eau, et à la compression dans le sens vertical sous l'action de leur propre poids, alors qu'avec un réservoir rectangulaire il y aurait eu des efforts de flexion. Pour diminuer l'évaporation et pour publiée par la Compagnie générale des Eaux de Paris qui a adopté le ciment armé pour plusieurs de ses ouvrages.

Excavations. - Ainsi que le montre la figure t, le réservoir est enterré assez profondément afin qu'il soit autant que possible à l'abri de la température extérieure. Le diamètre du réservoir étant de $36^{\mathrm{m}} 20$, on fit une excavation circulaire de $40^{\mathrm{m}}$ de diamètre ménageant un espace libre de Im8o nécessaire pour monter les échafaudages, installer les moules et faciliter les diverses minnceurres nécessaires. La profondeur moyenne de la fouille a été de $4^{\mathrm{m}} 7^{5}$ necessitant un mouvement de terre de $6230 \mathrm{~m}^{3}$. Jusqu'à $\mathrm{I}_{20}$ on a

COUPE VERTICALE ET ÉLEVATION
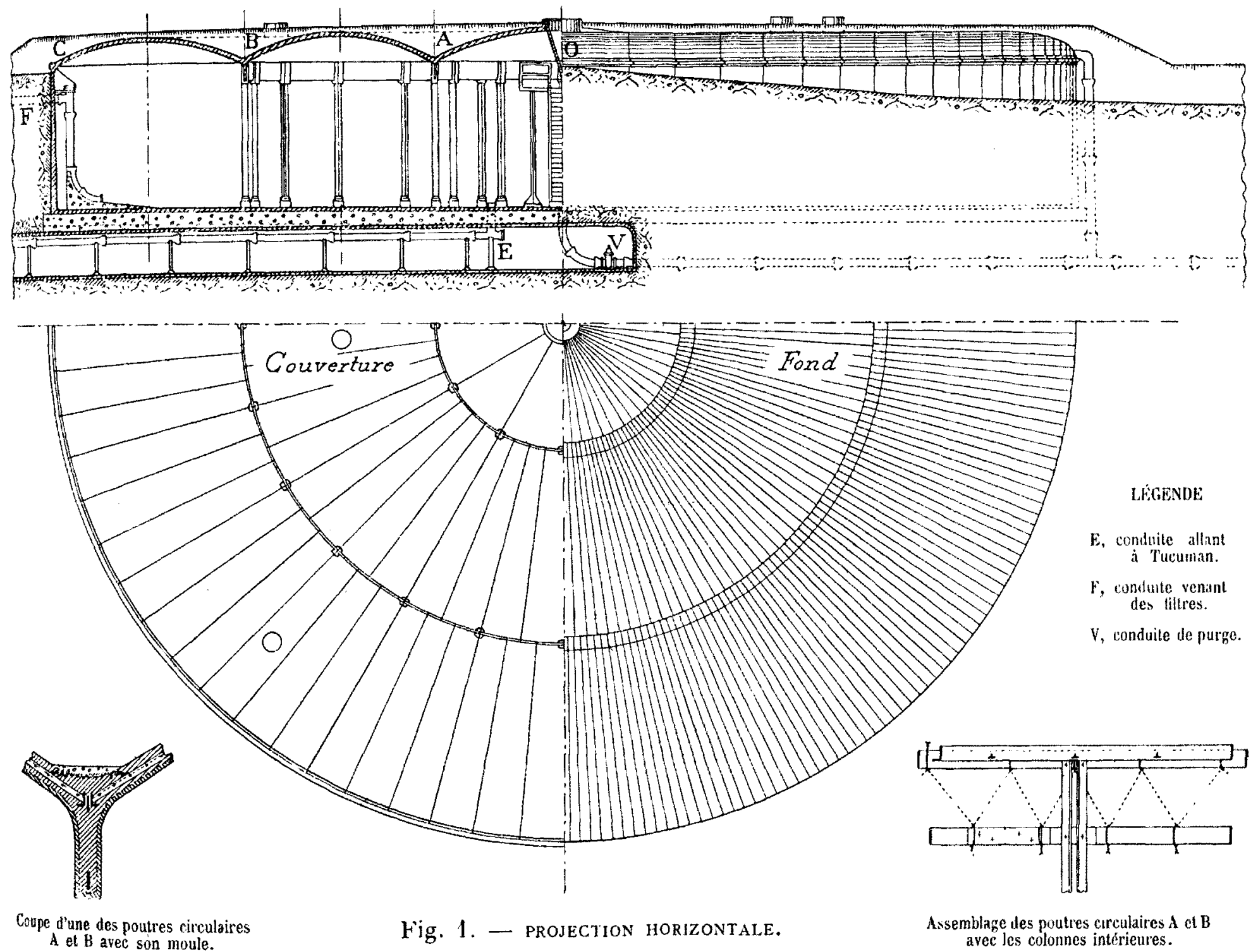

éviter l'échauffement de l'eau sous l'action d'un soleil tropical, on a muni le réservoir d'un toit, également en ciment armé, et recouvert lui-même d'une couche de terre minimum de $\mathrm{o}^{\mathrm{m}_{1}} \mathrm{o}$. Comme on le verra plus loin, ce toit est supporté pardeux séries de colonnes s'appuyant sur le fond duréservoir.

Pour avoir le minimum de surface extérieure, il aurait fallu que la hauteur fût égale au diamètre, mais si cette disposition est pratique pour les petites capacités il n'en est plus de même pour les grandes, aussi a-t-on limité la hauteur de l'eau à $5 \mathrm{~m}_{2} 5$ en employant un diamètre de $36^{\mathrm{m}} 20$. Quant aux dispositions générales on s'est basé sur la note trouvé de la terre végétale ou un terrain assez meuble qu'on a mis de côté pour recouvrir plus tard le toit du réservoir; au-dessous le terrain a été assez résistant pour qu'on ait pu dresser les parois verticalement et sans talus. On a procédé également aux fouilles nécessaires pour loger les canalisations d'amenée et d'évacuation de l'eau, avec une chambre de manceuvre des vannes, ce qui représente un supplément dé i $520 \mathrm{~m}^{3}$. Le total des fouilles, soit $7750 \mathrm{~m}^{3}$, a exigé $\mathrm{I} 84^{\circ}$ journées de so heures, soit une moyenne de $4 \mathrm{~m}^{3} 2$, par jour. Les déblais ont été utilisés au nivellement des terrains voisins.

Canalisations. - L'eau d'alimentation de la ville de 
Tucuman peut être prise soit dans le réservoir, soit directement aux filtres en cas de réparations au premier; un système de vannes réunies dans une chambre de manceuvre a été prévu à cet effet. Le réservoir est muni de deux orifices d'évacuation de trop plein; l'un est à la partie supérieure, l'autre au contraire est tout à fait à la partie inférieure, au centre du réservoir. La prise d'eau de la canalisation d'alimentation de la ville se trouve en $\mathrm{E}$ à $2 \mathrm{~m} 50$ du centre et à $\mathrm{o}^{\mathrm{m}}$ to au-dessus du fond du réservoir; de cette façon les terres et sables fins qui ont passé à travers les filtres peuvent se déposer en dessous de la prise d'eau. Le fond du réservoir est légèrement incliné depuis les parois jusqu'au centre, et la dénivellation est de $\mathrm{om}_{\mathrm{m}} \mathrm{o}$; l'une desprises d'eau de trop plein se trouvant juste au milieu du réservoir et au point le plus bas, il en résulte que si l'on ouvre la vanne $V$ que l'on voit au bas de la coupe verticale de la figure $I$, on provoque une chasse énergique de toutes les impuretés qui se sont amassées au fond du réservoir. L'amenée de l'eau venant des filtres se fait en $F$.

Afin de pouvoir vérifier à chaque instant l'état de la conduite amenant l'eau à la ville, on l'a logée dans une galerie de dimensions telles qu'un homme puisse y circuler facilement; elle a $\mathrm{I}^{\mathrm{m}} 50$ de hauteur sur $\mathrm{I}^{\mathrm{m}}$ de largeur et a été construite pour résister à une charge de 5250 kilos par mètre carré, la hauteur de l'eau dans le réservoir étant de $5 \mathrm{~m}_{2} 5$. Cette galerie a été faite en ciment armé; sa section est celle d'un rectangle surmonté d'un demi-cercle. Son armature métallique se compose de directrices verticales en fils de fer de $8 \mathrm{~m} / \mathrm{m}$ de diamètre, espacés de $8 \mathrm{~cm}$. les uns des autres et en génératrices horizontales, en fils de fer de $6 \mathrm{~m} / \mathrm{m}$, espacés de $7 \mathrm{~cm}$. et reliés aux précédents au moyen de ligatures en fil d'acier doux.

Fondations du réservoir. - Tout le fond de l'excavation a été recouvert d'une couche de béton de om 30 d'épaisseur dont la composition était la suivante: I partie, en volume, de ciment de Boulogne-sur-Mer (marque Demarle-Lanquety); 1 partie de sable fin (de moins de un demi-millimètre); 2,5 parties de sable moyen (de 0,5 à $2 \mathrm{~m} / \mathrm{m}$ ) et 4,5 parties de graviers ( 15 à $50 \mathrm{~m} / \mathrm{m}$ ).

Sur ce béton repose le fond proprement dit du réservoir. Ce fond est composé d'une triple rangée de petites poutrelles radiales noyées dans le ciment (voir le plan de la fig. I). Chaque rangée de poutrelles est solidement boulonnée à une armature circulaire qui la relie à la précédente ou à la suivante et sur laquelle viennent s'appuyer les colonnes circulaires qui supportent le toit. Il y a 57 poutrelles dans la première rangée intérieure, i i 4 dans la seconde et 228 dans la dernière qui touche aux parois. L'armature circulaire qui relie les poutrelles radiales et qui supporte la charge du toit, est formée d'une double couronne de poutrelles de

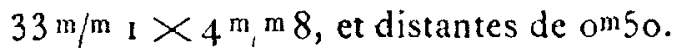

Parois verticales. - Le calcul de l'armature s'est fait en observant les prescriptions du service technique de la Compagnie générale des Eaux et en s'attachant à réaliser les conditions dans lesquelles $M$. Chassin s'est placé pour la construction du réservoir de $4000 \mathrm{~m}^{3}$ de Châtillon.

L'armature métallique des parois comprend (Voir fig. 2):

$I^{\circ}$ Une série de 21 directrices horizontales équidistantes verticalement de om 25 d'axe en axe, et dont la section va en croissant depuis le haut jusqu'en bas, conformément à la loi des pressions hydrostatiques. Si l'on appelle $h$, la hauteur de l'eau ali-dessus d'une des génératrices, $d$ le diamètre du réservoir, $l$ la distance des directrices d'axe en axe, $R=$ to $\mathrm{kgs}$ par $\mathrm{m} / \mathrm{m}^{2}$ le coefficient de résistance du fer et $m=3$ un coefficient empirique, la section $S$ de cette géne. ratrice doit être, d'après la formule employée par la Compagnie générale des Eaux:

$$
S=\frac{1000 h l d}{3 R}
$$

La tension variant de I $125 \mathrm{kgs}$ sur la première génératrice à 23625 sur la dernière, les sections des fers employés ont varié de $50,8 \times 3,2 \mathrm{~m} / \mathrm{m}$ à $139,7 \times 12,7 \mathrm{~m} / \mathrm{m}$, ce qui correspond à une charge de $7,5 \mathrm{kgs}$ par $\mathrm{m} / \mathrm{m}^{2}$ pour la première génératrice et de $13 \mathrm{~kg}$. 5 pour la dernière.

Le poids total de ces directrices verticales a été de 18 ooo kilos, en y comprenant les couvre-joints et rivets, ce qui pour une circonférence de $13^{\mathrm{m}} 7 \mathrm{o}$, donne un poids de $158 \mathrm{kgs}$ par mètre courant.

La longueur de chaque poutrelle composant les directrices a varié de 6 à 8 mètres et la jonction de l'une à l'autre s'est faite avec boulons à la partie supéricure et rivets à la partie inférieure, avec un double couvre-joint.

$2^{\circ}$ Une séric de 228 génératrices ou montants verticaux en fer à U, équidistants de om5o d'axe en axe. Chacune de ces génératrices verticales correspond avec une des poutrelles horizontales et radiales, constituant l'armature du fond du réservoir, dans le voisinage des parois verticales, et avec laquelle eile est solidement boulonnée. Ces montants ont été ainsi disposés : 76 fers $\mathrm{U}_{1}$ de $50 \times 25 \times 7 \mathrm{~m} / \mathrm{m}$, soit une section de $575 \mathrm{~m} / \mathrm{m}^{2}$ et un poids de $4,5 \mathrm{kgs}$ par mètre, ont été placés tous les $1^{m} 50$; et entre eux on a intercalé 152 fers $\mathrm{U}_{2}$ de $40 \times 20 \times 5,5 \mathrm{~m} / \mathrm{m}$, soit une section de $437 \mathrm{~m} / \mathrm{m}^{2}$ et un poids de $3,5 \mathrm{kgs}$ par mètre.

30 Un réseau de fils de 6 à $8 \mathrm{~m} / \mathrm{m}$ de diamètre et de feuillards de $8 \mathrm{~cm}$. de côté, noués entre eux à chaque croisement. - L'épaisseur des parois dans lesquelles sont noyées toutes ces armatures est de 20 centimètres.

Le ciment fut coulé entre deux rangées de planches formant moule et par anneaux successifs de $o^{m} 20$ de hauteur fortement pilonnés. Le travail fut poussé aussi activement que possible et complètement achevé en 6 jours; 12 hommes étaient employés au pilonnage et 53 à la préparation du béton. Ce dernier se composait en volume : de I de ciment, 1 de sable fin et $I, 5$ de sable grossier. 36 heures après qu'on eut fini le remplissage, on retira le moule extérieur tout en conservant l'intérieur et l'on commença, 24 heures après, à passer un enduit grossier d'environ I centimètre d'épaisseur pour dresser la paroi extérieure. Puis on procéda immédiatement au remplissage du vide qui restait entre la paroi et le bord de la fouille.

Colonnes intérieures. - La toiture dù réservoir est forméc d'un dôme central ct d'une double voûte circulaire, le tout en ciment armé. Cette toiture supporte une surcharge de terre d'une épaisseur aux sommets des vouttes de om$^{\mathrm{I}} \mathrm{o}$. Cette terre n'a pas été nivelée, mais au contraire disposée suivant un léger cône; Ies naissances des roûtes sont bien disposées suivant un même plan horizontal, maís les flèches vont constamment en diminuant depuis.l'taxe 
jusqu'à lá paroi pour suivre la direction de la terre placée sur la toiture. Ces voûtes reposent d'une part en $C$ sur la paroi verticale et d'autre part en B et en A sur deux poutres circulaires en ciment armé qui, à leur tour, sont supportées par deux rangées de colonnes verticales également en ciment armé.

La poutre circulaire A supporte le poids du dòme central et une partie du poids de la voûte $A B$; la poutre circulaire $B$ supporte une partie de la voûte $A B$ et une partie de la voùte $B C$; l'autre partie de la voûte $B C$ repose sur la paroi verticale.

Voùte centrale. - Le poids de la voûte centrale et celui de sa surcharge de terre se calculent sans difficulté. Le rayon de la courbe d'intrados de la voûte est de $9^{\mathrm{m}} 7 \mathrm{o}$, l'épaisseur de cette voûte est de $\mathrm{om}_{1} \mathrm{o}$ et l'épaisseur de la terre au point culminant est également de $o^{\mathrm{m}} \mathrm{r}$, et l'on trouve pour poids total :

$$
\mathrm{P}_{0 \mathrm{~A}}=77500 \text { kilogrammes. }
$$

$$
\begin{gathered}
d l=\sqrt{1+\left(\frac{d y}{d x}\right)^{2}} d x=\frac{R_{1} d x}{\sqrt{R_{1}^{2}-(x-a)^{2}}} \\
d p_{1}=\frac{2 \pi \varepsilon \delta R_{1}}{p_{2}-p_{1}} \times \frac{x\left(p_{2}-x\right)}{\sqrt{R_{1}^{2}-(x-a)^{2}}} d x \\
d p_{2}=\frac{2 \pi \varepsilon \delta R_{1}}{p_{2}-p_{1}} \times \frac{x\left(x-p_{1}\right)}{\sqrt{R_{1}^{2}-(x-a)^{2}}} d x
\end{gathered}
$$

$R_{1}$ étant la moyenne des rayons des courbes d'intrados ct d'extrados.

L'action de la voûte $A B$ sur la poutre circulaire $A$ est la somme des poids $d p_{1}$, c'est-à-dire :

$$
\begin{gathered}
P_{1}=\frac{2 \pi \pm \delta R_{1}}{p_{2}-p_{1}} \int_{p_{1}}^{p^{2}} \frac{x\left(p_{2}-x\right)}{\sqrt{R_{1}^{2}-(x-a)^{2}}} d x \\
P_{1}=2 \pi \varepsilon \delta\left[\left(p_{2}-a-\frac{R_{1}^{2}}{2 a}\right) \frac{a \operatorname{arc} A B}{p_{2}-p_{1}}+\frac{R_{1}}{2} V \frac{R_{1}^{2}-\left(p_{2}-a\right)^{2}}{p_{1}}\right]
\end{gathered}
$$
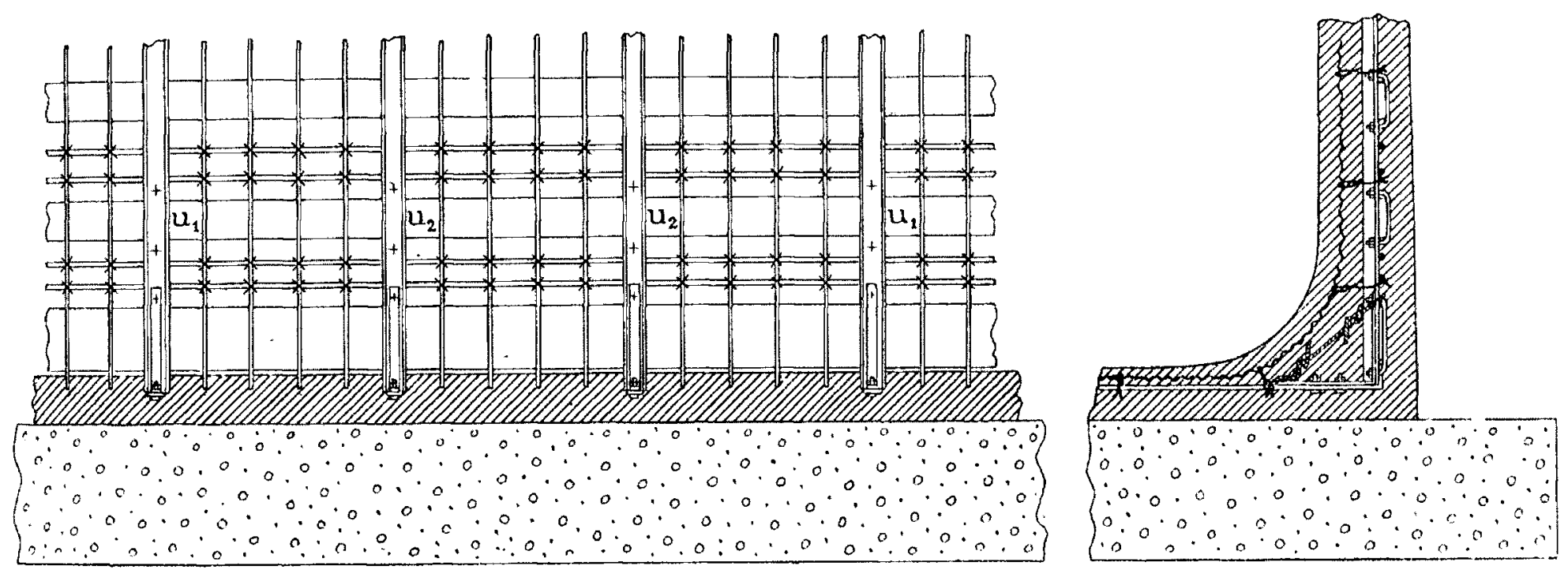

Fig. 2. - détails de l'armature de la parol Cylindrique.

Ce poids se répartit uniformément sur la poutre circulaire intérieure qui supporte en outre une partie de la charge de la voûte $\mathrm{AB}$.

Voutte circulaire intérieure. - Décomposons la voûte $\mathrm{AB}$ en une infinité de couronnes élémentaires de rayon $x$ et de longueur $: l l$. Le poids $d p$ de chacune de ces couronnes élémentaires sera égal à $2 \pi x \in \delta d l$, E étant l'épaisseur de la voûte et ò le poids du mètre cube de ciment armé; ce poids $d p$ peut être décomposé en deux autres $d p_{1}$ ct $d p_{2}$ agissant en $\mathrm{A}$ et en $\mathrm{B}$, et l'on a :

$$
d p_{1}=d p \frac{p_{2}-x}{p_{2}-p_{1}} \quad d p_{2}=d p \frac{\left(x-p_{1}\right)}{p_{2}-p_{1}}
$$

$p_{z}$ étant le rayon de la poutre circulaire $B$ et $p_{1}$ celui de la poutre A (Voir fig. 3).

Prenons deux axes de coordonnées rectangulaires $\mathrm{O}_{1} \mathrm{X}$ et $O_{1} Y$, l'axe $O_{1} Y$ passant par l'axe $O$ du réservoir, l'axe $O_{1} X$ passant par le centre $O^{\prime}$ de la voûte $A B$.

$$
\begin{array}{ll}
\text { Posons: } & a=\mathrm{O}_{1} \mathrm{O}^{\prime}=\frac{p_{1}+\mathrm{p}_{2}}{2} \\
\text { il vient : } & (x-a)^{2}+y^{2}=R_{1}^{2}
\end{array}
$$

L'action de la voûte $\mathrm{AB}$ sur la poutre circulaire $\mathrm{B}$ est également la somme des poids $d p_{\mathfrak{Q}}$ :

$$
\begin{gathered}
P_{2}=\frac{2 \pi \varepsilon \delta R_{1}}{p_{2}-p_{1}} \int_{p_{1}}^{p_{2}} \frac{x\left(x-p_{1}\right)}{\sqrt{R_{1}^{2}-(x-a)^{2}}} d x \\
P_{2}=2 \pi \approx \delta\left[\left(\frac{R_{1}^{2}}{2 a}+a-p_{1}\right) \frac{a \operatorname{arc} A B}{p_{2}-p_{1}}-\frac{R_{1}}{2} V \overline{R_{1}^{2}-\left(p_{2}-a\right)^{2}}\right]
\end{gathered}
$$

Comme vérification, si l'on fait la somme des charges $P_{1}$ et $P_{2}$ en $\mathrm{A}$ et $\mathrm{B}$, on trouve pour poids de la voûte $\mathrm{AB}$ :

$$
P=P_{4}+P_{2}=2 \pi a \times \approx \operatorname{arc} A B \times \delta
$$

que l'on aurait pu obtenir directement en remarquant que le volume engendré par une surface de révolution est égal au produit ce cette surface ( crite par le centre de gravité $(2 \pi a)$.

Décomposons également la surcharge de terre en une infinité de couronnes élémentaires de rayon $x$ et d'épaisseur $d x$. Le poids de chacune de ces couronnes élémentaires sera :

$$
d p=2 \pi x[h-y-(x-a) \operatorname{tg} \varphi] z^{\prime} d x
$$

o' étant le poids du mètre cube de terre. 
Comme précédemment il se décomposera en deux autres $d p_{1}$ et $d p_{2}$ agissant en $\mathrm{A}$ et en $\mathrm{B}$ :

$$
\begin{aligned}
& d p_{1}=2 \pi x[h-y-(x-a) \operatorname{tg} \phi] \delta^{\prime} \frac{p_{2}-x}{p_{2}-p_{1}} d x \\
& d p_{2}=2 \pi x[h-y-(x-a) \operatorname{tg} \varphi] \delta^{\prime} \frac{x-p_{1}}{p_{2}-p_{1}} d x
\end{aligned}
$$

$q$ étant l'angle que fait la surface externe de la terre, qui recouvre le toit, avec l'horizontale.

L'action de la surcharge de terre sur la poutre circulaire A sera par conséquent :

$$
\begin{aligned}
& P_{1}^{\prime}=\frac{2 \pi \partial^{\prime}}{p_{2}-p_{1}} \int_{P_{1}}^{p_{2}} x\left[h-V \overline{R_{2}^{2}-(x-a)^{2}}-(x-a) \operatorname{tg} \varphi_{i}\right]\left(p_{2}-x\right) d x \\
& =2 \pi \delta^{\prime}\left\{\begin{array}{l}
p_{2} a(h+a \operatorname{tg} q)-\frac{p_{2}^{3}-p_{1}^{3}}{3\left(p_{2}-p_{1}\right)}\left[h+\left(\hat{p}_{2}+a\right) \operatorname{tg} q\right] \\
+\frac{p_{2}^{4}-p_{1}^{4}}{4\left(p_{2}-p_{1}\right)} \operatorname{tg} q-\frac{1}{4}\left[R_{2}^{2}-\left(p_{2}-a\right)^{2}\right]^{3 / 2} \\
-\frac{a}{2}\left(p_{2}-a-\frac{R_{2}^{2}}{4 a}\right)\left(V \overline{R_{2}^{2}-\left(p_{2}-a\right)^{2}}+\frac{R_{2} \operatorname{arcdB}}{p_{2}-p_{1}}\right)
\end{array}\right.
\end{aligned}
$$

$R_{2}$ est ici le rayon de l'arc de cercle de l'extrados de la voûte $A B$.

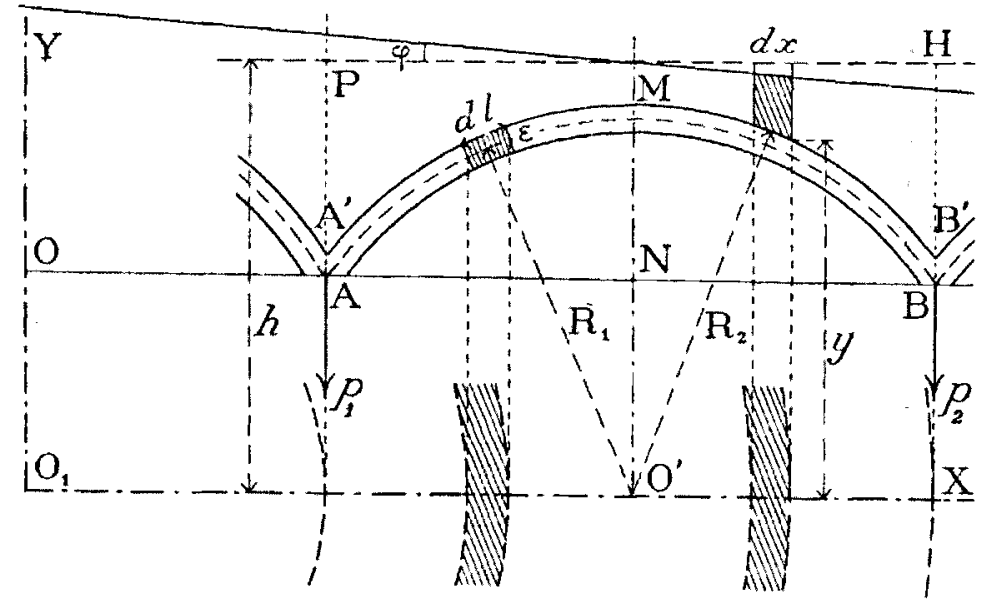

Fig. 3

De même l'action de la surcharge sur la poutre B sera :

$$
\begin{aligned}
& P_{2}^{\prime}=\frac{2 \pi \delta^{\prime}}{p_{2}-p_{1}} \int_{\rho_{1}}^{p_{2}} x\left[h-V \overline{R_{2}^{2}-(x-a)^{2}}-(x-a) \operatorname{tg} \varphi\right]\left(x-p_{1}\right) d x \\
& P_{2}^{\prime}=2 \pi \hat{o}^{\prime}\left\{\begin{array}{l}
\frac{p_{2}{ }^{3}-p_{1}{ }^{3}}{3\left(p_{2}-p_{1}\right)}\left[h+\left(p_{1}+a\right) \operatorname{tg} \varphi\right] \\
-p_{1} a(h+a \operatorname{tg} \varphi)-\frac{p_{2}{ }^{4}-p_{1}^{4}}{4\left(p_{2}-p_{1}\right.}+\frac{1}{4}\left[R_{2}{ }^{2}-\left(p_{2}-a\right)^{2}\right]^{3 / 2} \\
-\frac{a}{2}\left(\frac{R_{2}{ }^{2}}{4 a}+a-p_{1}\right)\left(V \overline{R_{2}{ }^{2}-\left(p_{2}-a\right)^{2}}+\frac{R_{2} \operatorname{arcAB}}{p_{2}-p_{1}}\right)
\end{array}\right.
\end{aligned}
$$

Comme précédemment, si l'on fait la somme des poids $P^{i}$ et $P_{2}^{\prime}$ agissant en $\mathrm{A}$ et $\mathrm{B}$ (en négligeant les termes en $\operatorname{tg} \varphi$, afin de faciliter la vérification, саг alors le centre de gravité se trouve sur la verticale passant par le centre O' de l'arc de cercle d'extrados), on retrouve le poids que l'on aurait obtenu directement en faisant le produit de la surface A'M B' H P par la circonférence que décrit le centre de gravité:

$$
P=2 \pi a \delta^{\prime}\left\{\begin{array}{c}
P=P_{1}^{\prime}+P_{2}^{\prime} \\
\left(p_{2}-p_{1}\right)\left(h-\frac{\sqrt{R_{2}^{2}-\left(p_{2}-a\right)^{2}}}{2}\right) \\
-\frac{1}{2} R_{2} \operatorname{arc} \mathrm{AB}
\end{array}\right.
$$

Or l'on a : $p_{1}=\mathrm{OA}=4^{\mathrm{m} 50} ; p_{2}=\mathrm{OB}=\mathrm{I}_{1}^{\mathrm{m}} \mathrm{m}_{2}$; $p_{2}-p_{1}=A B=6^{m} 7^{5} ; O_{1} O^{\prime}=a=\frac{1}{2}\left(p_{1}+p_{2}\right)=7^{m} 875 ;$ $R_{1}=6 \mathrm{~m} 35 ; R_{9}=6^{\mathrm{m}} 4 \mathrm{o} ; h=6 \mathrm{~m}^{\mathrm{m}} 5 \mathrm{o} ; \varepsilon=\mathrm{o}_{\mathrm{m}} ; \delta=2500 \mathrm{~h}$; $\hat{s}^{\prime}=1500 \mathrm{k} . ; \mathrm{tg} q$ est déterminée par ce fait que la différence de hauteur entre la flèche de l'arc central OA et celle du dernier arc $\mathrm{BC}$ est de $\mathrm{o}^{\mathrm{m}} 22$ pour une distance horizontale de $14^{\mathrm{m}} 675$. Ces valeurs donnent :

$$
\begin{aligned}
P_{1} & =37500 \mathrm{kilog} . & P_{2} & =51000 \mathrm{kilog} . \\
P_{1}^{\prime} & =94000 \mathrm{~N} & P_{2}^{\prime} & =108500 \\
P_{1}+P_{1}^{\prime} & =131500 \mathrm{kilog} . & P_{2}+P_{2}^{\prime} & =159500 \mathrm{kilog} .
\end{aligned}
$$

Voûte circulaire extérieure.- Cette dernière voûte BC étant en tout semblable à la première, les charges en $B$ et $C$ se déterminent exactement de la même façon, $\operatorname{tg} q, \varepsilon, \partial$ et $\partial^{\prime}$ ont la même valeur que précédemment, mais il faut faire: $p_{1}=\mathrm{OB}=1 \mathrm{I}^{\mathrm{m}} 25 ; p_{2}=\mathrm{OC}=18^{\mathrm{m}} 10 ; p_{2}-p_{1}=\mathrm{BC}=6 \mathrm{~m} 85 ;$ $\mathrm{O}_{1} \mathrm{O}^{\prime}=a=14^{\mathrm{m}} 67^{\mathrm{m}} ; R_{1}=7^{\mathrm{m} 55} ; R_{\mathrm{q}}=7^{\mathrm{m} 60} ; h=7^{\mathrm{m}} 70_{\mathrm{i}}$ ce qui donne alors :

$$
\begin{array}{rr}
P_{1}=75000 & P_{2}=88000 \\
P_{1}^{\prime}=\frac{147000}{P_{2}^{\prime}}=170000 \\
P_{1}+P_{1}^{\prime}=22000 & P_{2}+P_{2}^{\prime}=258000
\end{array}
$$

Il en résulte que la charge de la poutre circulaire A est: $77500+131500=209000$ kilos qui, répartis sur une circonférence de $28^{\mathrm{m}} 27$, donne une charge de 7400 kilos par mètre courant. Comme cette poutre circulaire A est supportée par 12 colonnes, chacune d'elles a à supporter 17400 kilos. La section de cette colonne étant de 575 centimètres carrés, cela fait 3o kilos par centimetres carrés pour le travail du béton, en admettant que le fer ne soit là que pour s'opposer à la flexion.

La charge sur la seconde poutre circulaire B est:

$$
159500+222000=381500 \mathrm{kgs}
$$

qui, répartis sur une circonférence de $70^{\mathrm{m} 69}$, donne une charge de 5400 kilos par mètre courant. Cette poutre estsupportée par 24 colonnes distantes de $2^{\mathrm{m}} 94$, qui ont cha. cune à supporter 15 goo kgs. La section de ces colonnes étant comme précédemment de $575 \mathrm{~cm}^{2}$, le béton a à supporter une pression de $27 \mathrm{kgs} 6$ par $\mathrm{cm}^{2}$.

Les 36 colonnes qui supportent la toiture du réservoir sont toutes faites sur le même modèle (voir figure 4). Leur section est celle d'un carré de $\mathrm{o}^{\mathrm{m}} \mathbf{2} 5$ de côté dont on aurạit abattu les angles. A leur intérieur sont noyés 4 fers corniè res, de $50 \times 50 \times 6 \mathrm{~m} / \mathrm{m}$, distants de $3 \mathrm{~cm}$. et reliés entre eux par des entretoises boulonnées. Pour augmenter la surface de base, le côté du carré primitif de leur section est porté à om8o. De plus ces 4 fers cornières verticaux sont reliés à leur base à 2 autres fers cornières horizontaux de même section et d'une longueur de om8o. Les fers verticaux et horizontaux sont en outre entretoisés au moyen de 4 fers 
en U de $40 \times 20 \times 5,5 \mathrm{~m} / \mathrm{m}$ disposés dans deux plans perpendiculaires. Pour assurer encore une meilleure répartition de la charge sur le fond du réservoir et par là éviter la formation de fissures, on a disposé sur chaque colonne un carré de om8o de côté formé d'un treillis de fils de fer de $8^{m} / \mathrm{m}$ de diamètre et enrobé dans le ciment.

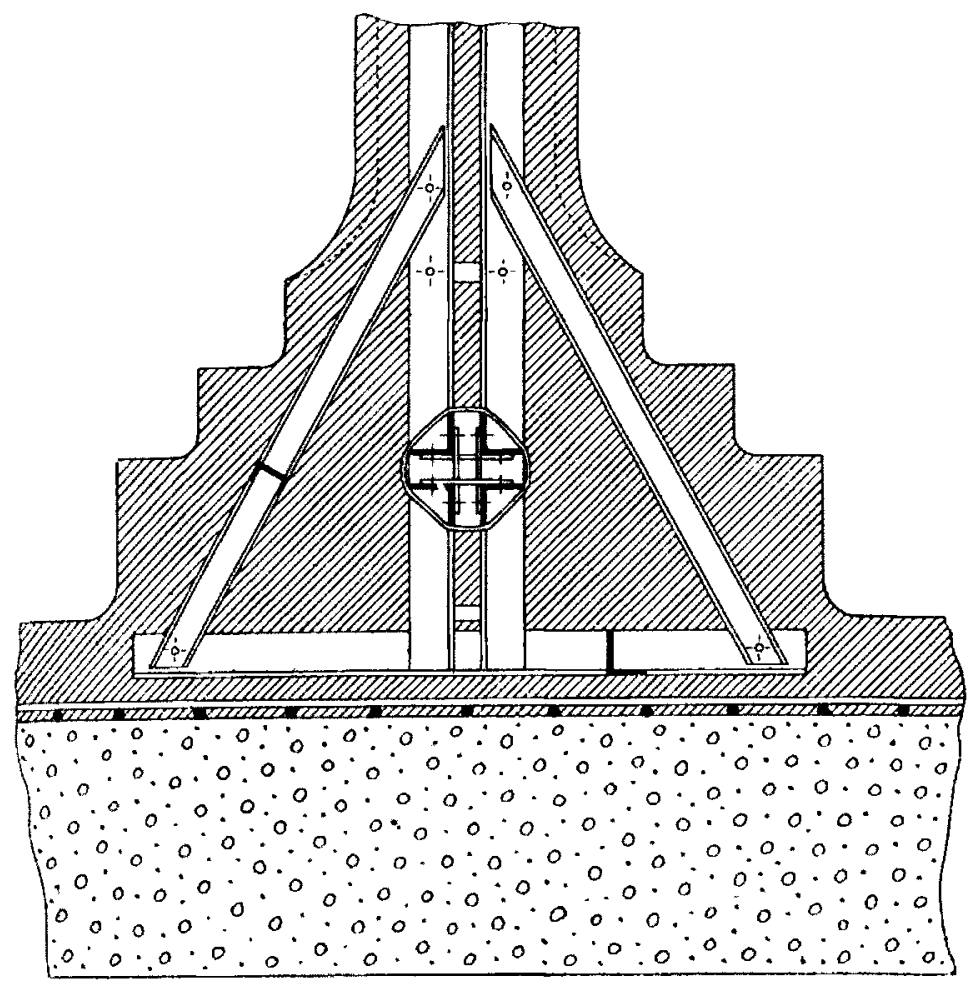

Fig. 4. - ASSISE de l'une des COlONNES intÉrieures.

Le béton de ces colonnes est composé de: I partie de ciment, $\mathrm{I}, 5$ de sable fin et $\mathrm{I}, 5$ de sable grossier; un ouvrier mettait 5 heures pour garnir un moule formant une colonne.

Poutres armées circulaires. - Ces poutres qui reposent sur les colonnes précédemment étudiées sont formées de deux poutrelles métalliques semblables, supérieure et inférieure, équidistantes du centre de la poutre et reliées entre elles par des fils de fer; le tout étant noyé dans le ciment.

Ces poutres ont été calculées comme s'il s'agissàit de poutres séparées encastrées à leurs deux extrémités; dans ce cas on a pour la poutre circulaire $A$, chacune de ses colonnes étaut espacée de $2^{\mathrm{m}} 35$ :

$$
M=\frac{P l^{2}}{12}=\frac{7400 \times\left(2^{\mathrm{m} 35}\right)^{2}}{12}=3400 \text { kilogrammètres. }
$$

Si l'on suppose que le fer travaille seul on doit avoir, d'après la formule de M. Lefort:

$$
R \times \frac{I}{n}=M=R \times S \times h
$$

$S$ étant la section de l'une des armatures et $h$ la distance des centres de gravité de celles-ci.

Or les armatures de la poutre circulaire sont des fers plats de $95,2 \times 125 \mathrm{~m} / \mathrm{m}$ représentant une section de $120 \mathrm{~g}^{\mathrm{m}} / \mathrm{mz}$; il s'en suit que la tension du métal est :

$$
R=\frac{M}{S h}=\frac{3,400}{0,4 \times 0,001209}=7030000 \mathrm{kgs} \text { par } \mathrm{m}^{2}
$$

soit 7 kilos par $\mathrm{m} / \mathrm{m}^{2}$.
Pour la poutre $B$ on a :

$$
\begin{gathered}
M=\frac{P l^{2}}{12}=\frac{5400 \times\left(2^{\mathrm{m}_{94}}\right)^{2}}{12}=3900 \text { kilogrammetres } \\
R=\frac{3900}{0,4 \times 0,001209}=\text { soit } 8 \mathrm{kgs} \mathrm{par} \mathrm{m} / \mathrm{m}^{2}
\end{gathered}
$$

La poussée des voûtes n'étant pas la même de chaque côté de la poutre, celle-ci est soumise à un effort de renversement. Pour lui résister on a muni la partie supérieure de la poutre de deux cornières de $76 \times 50 \times 8 \mathrm{~m} / \mathrm{m}$. (Voir au bas de la fig. I les détails de construction de ces poutres).

Voûtes de la toiture. - Les voûtes de la toiture ont une épaisseur de $o^{m} 10$. Elles sont constituées par un ensemble de génératrices radiales et courbes formées de fers a $T$ de $38, \mathrm{r} \times 38, \mathrm{r} \times 4,8 \mathrm{~m} / \mathrm{m}$, du poids de 2,5 kilos par mètre linéaire et au nombre de 37 pour la coupole centrale, de 94 pour la voûte intérieure $A B$ et de 152 pour la voùte extérieure $\mathrm{BC}$.

Ces fers à $T$ sont croisés par un ensemble de fils de fer de $8 \mathrm{~m} / \mathrm{m}$ de diamètre espacés de 10 centimètres les uns des autres. Le béton est constitué par parties égales de sable fin et de ciment

Dispositions accessoires. - La jonction de la paroi verticale et du fond a été l'objet de soins tout particuliers, car c'est là un point faible où des fissures peuvent se produire le plus facilement sous l'action d'une flexion des parois. - Pour résister à la poussée de la voûte circulaire extérieure $\mathrm{BC}$, on a muni la paroi verticale cylindrique du réservoir de fers en $U$ de $140 \times 60 \times 7 \mathrm{~m} / \mathrm{m}$ reliés entre eux par une ceinture de tôle de $140 \times 16$ millimètres.

Lorsque tous les échafaudages furent enlevés on procéda au repiquage du fond et des parois, pour les dresser et les nettoyer, puis on y mit un enduit de mortier composé de I partie de ciment, I de sable fin et 2 de sable grossier, sur une épaisseur de 2,5 centimètres au fond et 0,5 centimètre en haut des parois. Par dessus on passa un badigeon de ciment pur.

A la clé des voûtes on a ménagé des ouvertures pour renouveler l'air intérieur. Au centre de la coupole intérieure on en a ménagé une beaucoup plus large. Pour permettre une visite facile du réservoir cette ouverture donne sur une petite plateforme centrale de $3 \mathrm{~m}$ de diamètre qui est supportée par 4 colonnes de $4^{\mathrm{m}} 50$ de hauteur et de $150 \mathrm{~cm}^{2}$ de section. De cette plateforme on peut descendre au fond du réservoir par un escalier également en béton armé et incliné de $35^{\circ}$ environ.

Il sera intéressant de connaître d'ici quelque temps les résultats donnés par ce travail, au point de vue de l'élasticité et de l'étanchéité des parois. Ces résultats sont du plus haut intérêt pour nos travaux d'aménagement des forces hydrauliques oú le béton armé joue de plus en plus un rôle prépondérant pour les canalisations à grand diamètre. Ces ouvrages sont évidemment bealucoup plus économiques de construction que par l'emploi des maçonneries ou des tôles d'acier; leur application rationnelle doit se répandre; reste à savoir si l'expérience vérifiera les prévisions sur lesquelles est fondée leur généralisation à un grand nombre de travaux d'art. L'avenir pourra nous le dire à brève échéance.
H. BELLET. 